\title{
Large-Scale Molecular Dynamics and High-Resolution Transmission Electron Microscopy Study of Graphene Grain Boundaries
}

\author{
Colin Ophus ${ }^{1}$, Haider Rasool ${ }^{2,3}$, Alex Zettl $^{2,3}$, and Ashivni Shekhawat ${ }^{3}$ \\ 1. National Center for Electron Microscopy, Molecular Foundry, Lawrence Berkeley National \\ Laboratory, Berkeley, USA
}

2. Department of Physics, University of California Berkeley, Berkeley, USA

3. Materials Science Department, Lawrence Berkeley National Laboratory, Berkeley, USA

Graphene is a promising material for various technological applications, due to its excellent electrical and [1] mechanical properties [2]. New graphene deposition methods are continually increasing the maximum fabricated sheet size of a single crystallographic orientation, but most deposition methods still produce polycrystalline sheets, containing grain boundaries (GBs) [3]. Graphene GBs are very interesting scientifically due to the two-dimensional nature of graphene. Bulk three-dimensional materials require 5 angles to characterize the macroscopic degrees of freedom, while two-dimensional materials require only 2 angles: the misorientation angle $\theta_{\mathrm{M}}$, defined as the angle between the unit cell vectors of each grain, and the boundary line direction $\theta_{\mathrm{L}}$, defined as the angle between the boundary vector and the symmetric tilt boundary vector. Thus the parameter space for 2D GBs is far smaller than that of 3D grain boundaries. With modern computer simulation methods, it is now possible to enumerate this entire parameter space in a very fine-grained manner.

In this study, we have combined phase-contrast high resolution transmission electron microscopy (HRTEM) observations of free-standing graphene GBs with molecular dynamics (MD) simulations spanning the entire orientation parameter space of graphene GBs. We have collected hundreds of observations of GB structure using HRTEM images, and dozens of higher-precision measurements using complex exit wave reconstruction (EWR) of HRTEM focal series [4]. Figure 1 shows lattice strain measurements of a graphene GB performed on an EWR dataset taken from our study in [5].

The experimentally-determined structures are compared to over $40000 \mathrm{MD}$ simulations of graphene GB structures, with steps of $0.5^{\circ}$ over $\theta_{\mathrm{M}}$ and $\theta_{\mathrm{L}}$. The structures were generated by fist constructing rational approximates of tiled graphene cells with the desired grain angles, periodic along the boundary line. Next the atomic coordinates were updated using a simple geometric algorithm to generate plausible sp2bonded structures. Finally the structures were relaxed using molecular dynamics. Figure 2 shows a subset of the calculated structures. From these simulated boundaries, we can evaluate many physical and energetic parameters; some examples are given in Figure 3. We can see that the GB enthalpy is very closely related to the dislocation density of the GB. In this talk we will examine various measurable parameters and compare them directly to experiment, with the aim of creating a predictive model of graphene GB structure, stability and resistance to deformation or fracture.

\section{References}

[1] OV Yazyev and SG Louie, Nature Materials 9 (2010) p. 806

[2] Y Wei, J Wu, H Yin, X Shi, R Yang and M Dresselhaus, Nature Materials 11 (2012) p. 759

[3] Haider deposition paper.

[4] H Rasool, C Ophus, WS Klug, A Zettl and JK Gimzewski, Nature Comm. 4 (2013) p. 2811

[5] HI Rasool, C Ophus, Z Xiang, MF Crommie, BI Yakobson, and A Zettl, Nanoletters 14 (2014) p. 7057

[6] The authors acknowledge the financial support of the Office of Science, Office of Basic Energy

Sciences of the US Department of Energy under contract number De-AC02-05CH11231. 

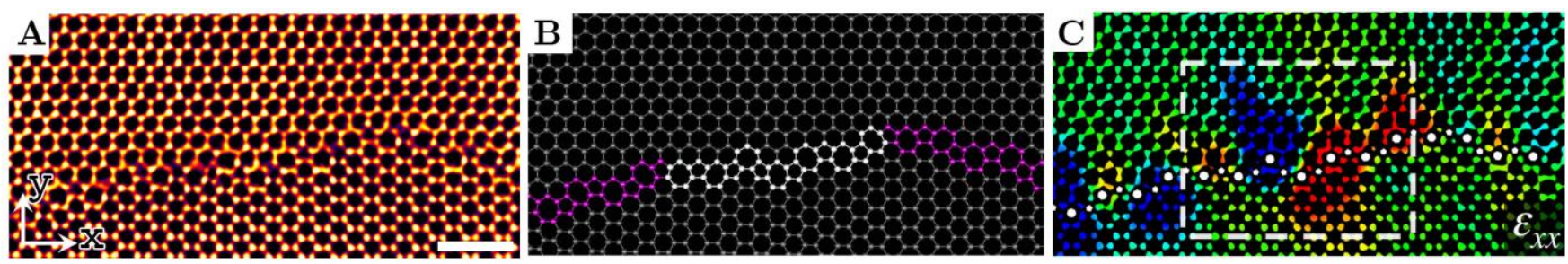

Figure 1. EWR image reconstructed from HRTEM focal series (A), showing the structure of a high angle graphene GB (B). Strain along $\mathrm{x}$-axis shown from $-1 \%$ (blue) to $+1 \%$ (red) plotted in (C). Scale bar length is $1 \mathrm{~nm}$, data and analysis in this figure is from [5].

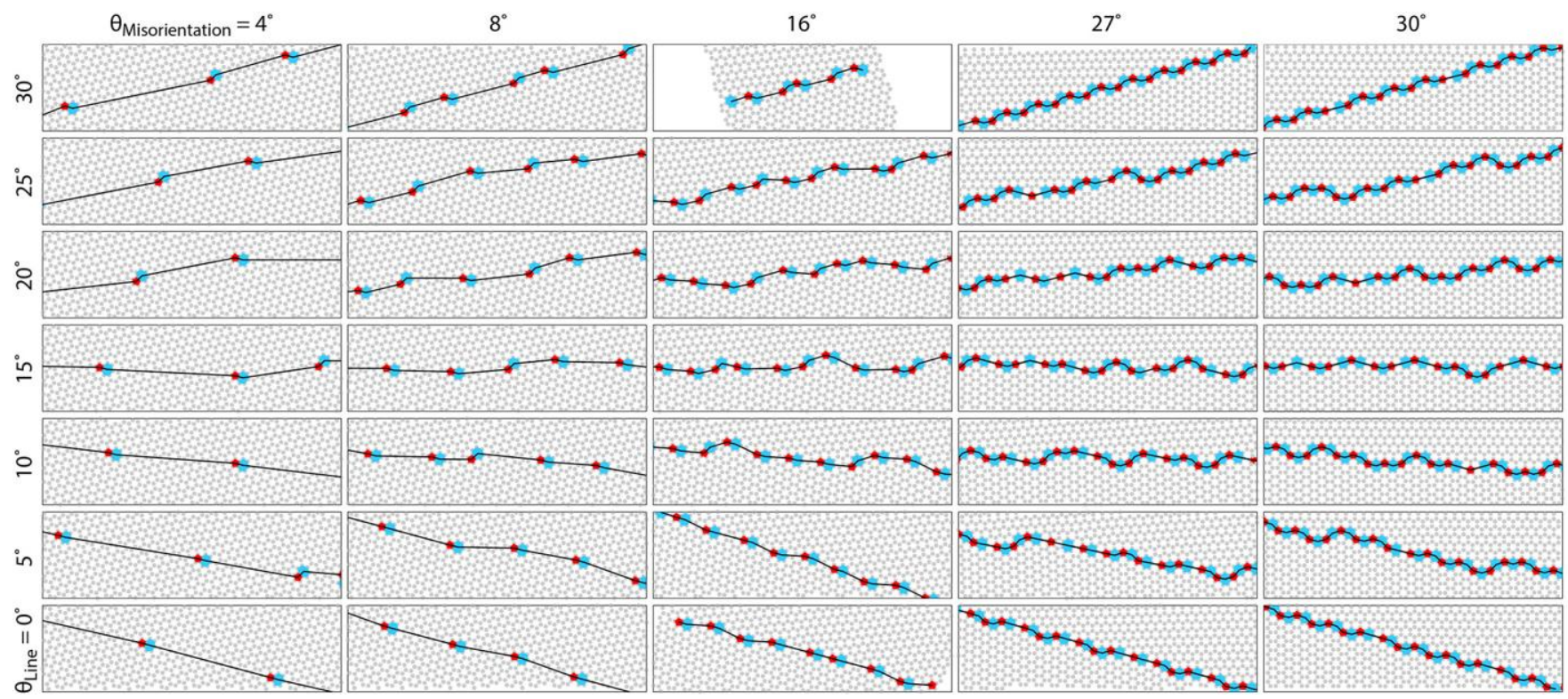

Figure 2. Graphene grain boundaries computed with molecular dynamics for misorientation angles of $4,8,16,27$, and 30, and boundary line angles of $0,5,10,15,25$ and 30. Red pentagons and blue heptagons show 5-atom and 7-atom carbon rings respectively, black line traces boundary.
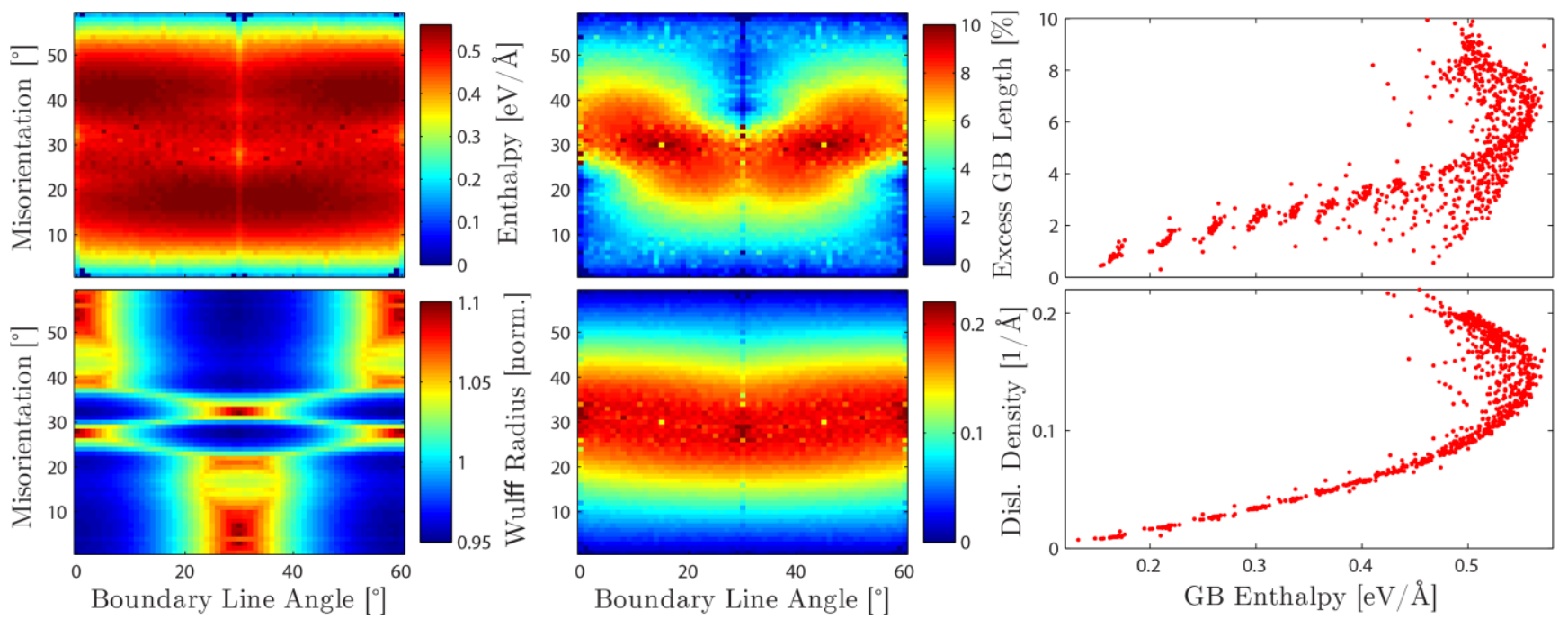

Figure 3. Measurements of graphene GB enthalpy, Wulff shape, excess length, and dislocation density simulated with MD. Enthalpy vs dislocation density and excess length are compared. 